J3eA - Vol. 3 - 5 (2004).

DOI : 10.1051/bib-j3ea:2004005

\title{
Récupération de porteuse dans les systèmes de transmissions analogiques et numériques
}

\author{
C. Chatellier ${ }^{*}$ "et J. Brochard ${ }^{* *}$ (Université de Poitiers) \\ Mis en ligne le 18/06/2004.
}

\begin{abstract}
Résumé
Nous présentons une maquette didactique permettant l'étude d'une boucle à verrouillage de phase ainsi qu'une boucle de Costas utilisées dans les systèmes de transmissions numériques. Après une présentation succincte de deux types de modulations numériques ASK (Amplitude Shift Keying) et PSK (Phase Shift Keying), nous abordons le problème de la démodulation synchrone à partir de considérations sur la stabilité d'une PLL (Phase Lock Loop). Nous appliquons alors les résultats de cette étude à l'utilisation d'une boucle de Costas pour la démodulation d'un signal PSK-2. Enfin nous donnons tous les éléments nécessaires à la réalisation d'une maquette avec quelques résultats ainsi que le contenu du TP associé.
\end{abstract}

Mots-clés : modulations numériques, boucle à verrouillage de phase, boucle de Costas, stabilité.

(C) EDP Sciences, 2004.

Niveau de connaissances requis. Une bonne connaissance de la PLL et des modulations.
Niveau des étudiants. Deuxième cycle, licence, maîtrise EEA, préparation à l'agrégation de physique appliquée.

* Christian Chatellier ${ }^{1}$ a obtenu le doctorat en sciences spécialité électronique de l'Université de Poitiers en février 1993. Depuis 1994 il occupe un poste de maître de conférences à l'Université de Poitiers où il enseigne l'électronique analogique et les communications numériques en licence et en maîtrise EEA, ainsi que dans le DESS Ingénierie des Systèmes de Transmission et de Communication (ISyTeC) dont il est le responsable.

Ses activités de recherche au sein l'équipe Signal Image et Communication de l'IRCOM (Institut de Radiocommunication Optique et Micro-onde) concernent l'étude et la simulation des systèmes de communications numériques.

o e-mail : chatellier@sic.sp2mi.univ-poitiers.fr

** Jacques Brochard ${ }^{1}$ a tout d'abord enseigné l'électronique et l'électrotechnique dans les lycées, en qualité de professeur agrégé de physique appliquée. Depuis 1984, il occupe un poste de maître de conférences à l'Université de Poitiers, où il enseigne l'électronique et l'automatique, essentiellement en maîtrise EEA et dans la préparation à l'agrégation de physique appliquée dont il est le responsable.

Ses activités de recherche au sein de l'équipe Signal Image et Communication de l'IRCOM concernent l'analyse d'images texturées statiques et dynamiques.

\footnotetext{
${ }^{1}$ Laboratoire IRCOM-SIC, Bâtiment SP2MI, Boulevard Marie et Pierre Curie - Teleport 2, BP 30179, F-86962 Futuroscope Chasseneuil Cedex, France.
} 


\section{Introduction}

La grande majorité des démodulateurs de signaux analogiques ou numériques fonctionnent suivant le principe de démodulation cohérente. Il s'agit de multiplier le signal modulé reçu par un signal qui présente les mêmes caractéristiques (fréquence et phase) que l'onde porteuse. Quelques équations simples montrent alors que l'information est extraite après un filtrage de type passe-bas.

Dans la plupart des ouvrages traitant des démodulations, on suppose disposer d'un oscillateur local calé en phase et en fréquence avec le signal porteur. Dans la réalité, ce signal n'est en général pas contenu dans le spectre du signal reçu. On doit donc recourir à l'élaboration de systèmes analogiques particuliers pour récupérer cette information indispensable à l'application du principe de démodulation cohérente.

Cet article présente une maquette didactique qui permet d'étudier les techniques, en général peu traitées et pourtant indispensables, utilisées en récupération de porteuses. Cette maquette peut donc servir de support à un TP d'électronique en licence ou en maîtrise EEA sur la PLL et la boucle de Costas.

\section{Modulations numériques}

Le but de ce paragraphe n'est pas de faire un cours sur les modulations numériques, mais plutôt de comprendre comment elles s'écrivent. Par rapport aux modulations analogiques, les modulations numériques ont un nombre fini d'états $M$ qui est en général égal à une puissance de 2. On parle alors de modulations $M$-aires, où $M=2^{\mathrm{n}}$ et $\mathrm{n}$ est le nombre de bits correspondant à un état.

Chaque série de $n$ bits est appelée symbole, et le débit symboles également appelé rapidité de modulation s'exprime en Bauds. Ainsi dans le cas d'une modulation 4-aires, si le débit binaire de la source est de $1 \mathrm{Mbits} / \mathrm{s}$, la rapidité de modulation sera de $500 \mathrm{kBauds}$. De la même façon, si $\mathrm{T}_{\mathrm{b}}$ est le temps d'émission d'un bit par la source, le temps symbole sera alors $\mathrm{T}=\mathrm{nT}_{\mathrm{b}}$.

\subsection{Modulation d'amplitude MDA - Amplitude Shift Keying (ASK)}

La modulation d'amplitude s'écrit :

$$
x(t) \equiv \sum_{k} a_{k} h(t-k T) \cos \left(2 \pi f_{0} t\right)
$$

Le symbole transmis $a_{k}$ prend sa valeur dans l'alphabet de M éléments

$$
\left\{A_{0}, A_{1, \ldots,}, A_{M-1}\right\}
$$
exemple de modulation d'amplitude binaire (modulation On Off Keying), la figure 2 montre un exemple de modulation d'amplitude quaternaire (ASK-4) avec leurs dictionnaires associés. 


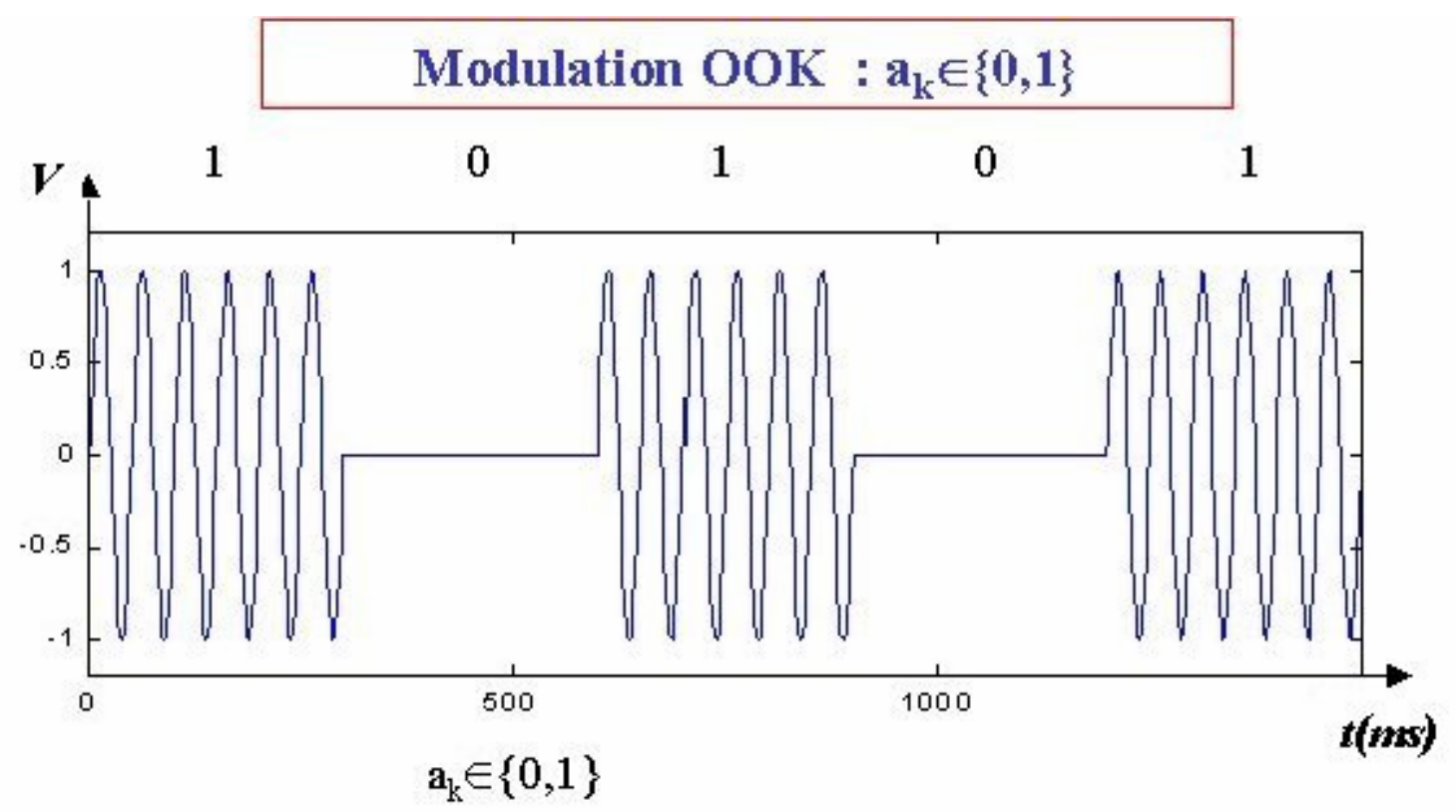

Fig. 1. Modulation d'amplitude OOK (On Off Keying).

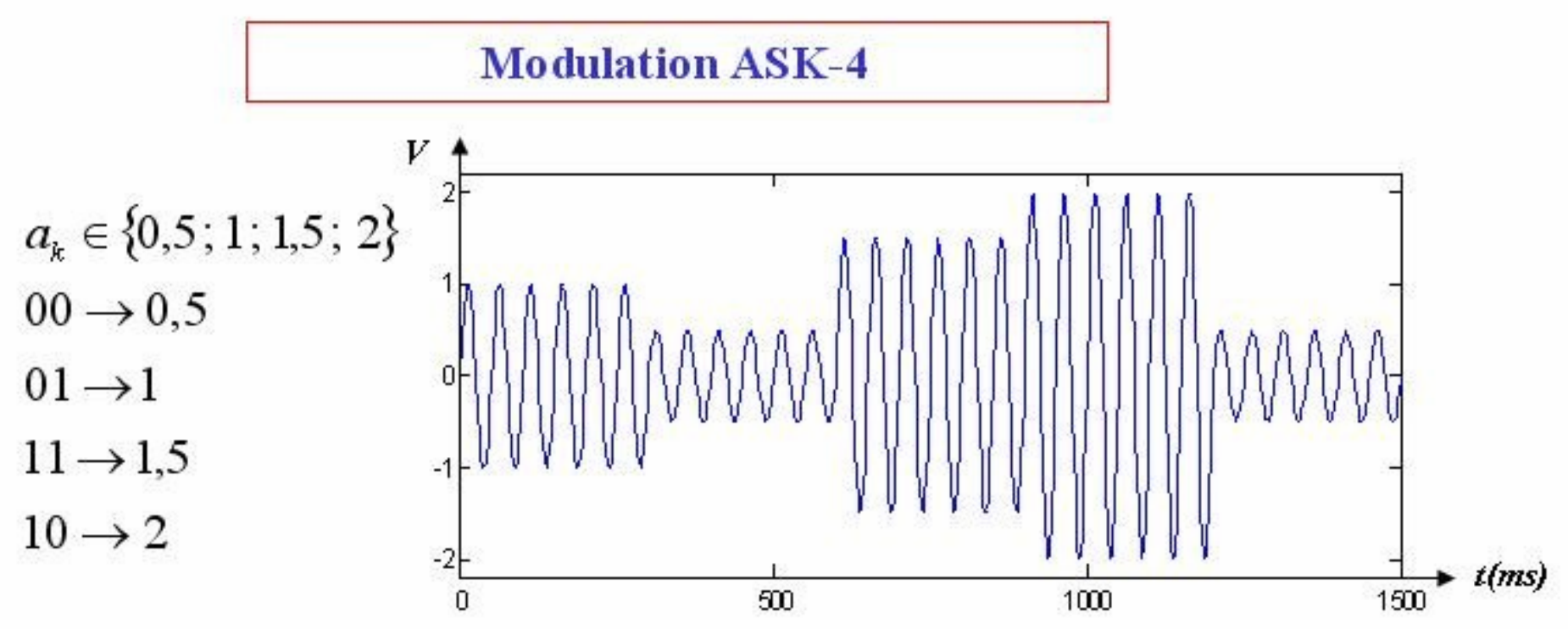

Fig. 2. Modulation d'amplitude ASK-4 et dictionnaire associé.

On peut également représenter géométriquement les différents états du signal modulé dans le plan de Fresnel pour obtenir des figures appelées constellations. Les constellations des signaux précédents sont représentées sur la figure 3. 


\section{Modulation ASK}

OOK

ASK-4
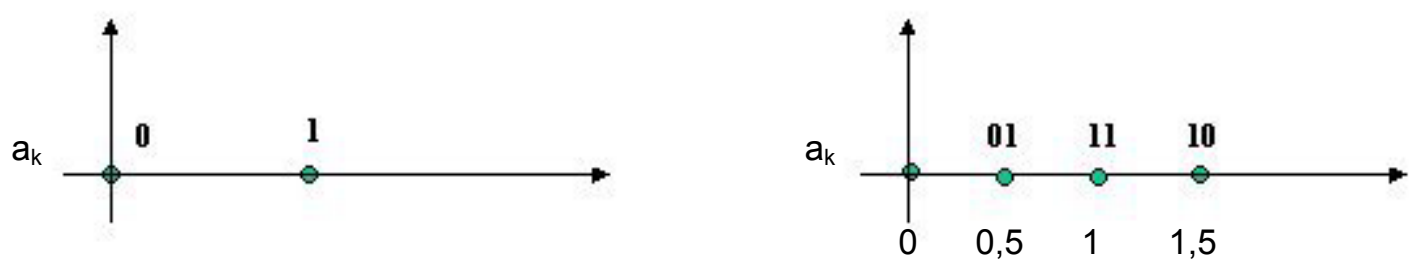

Fig. 3. Exemples de constellations en modulation d'amplitude.

\subsection{Modulation de Phase - Phase Shift Keying (PSK)}

La modulation de phase (Phase Shift Keying) s'écrit :

$$
x(t)=A \sum_{k} h(t-k T) \cos \left(2 \pi f_{0} t+\phi_{k}\right)
$$

Un symbole transmis est représenté par une phase ${ }^{\phi_{k}}$ qui prend sa valeur dans l'alphabet de M éléments $\left\{\phi_{0}, \phi_{1}, \ldots, \phi_{M-1}\right\}$

. C'est une modulation à amplitude constante dont les points de la constellation se situent sur un cercle. Un exemple de modulation PSK-4 et la constellation associée sont représentés sur la figure 4.

Il existe une autre écriture de cette modulation, en effet si on développe la fonction cosinus dans l'expression de $\mathrm{x}(\mathrm{t})$, on obtient :

$$
x(t)=A \sum_{k}\left[\cos \left(\phi_{k}\right) \cos \left(\omega_{0} t\right)-\sin \left(\phi_{k}\right) \sin \left(\omega_{0} t\right)\right] h(t-k T)
$$

Le signal $\mathrm{x}(\mathrm{t})$ se présente donc sous la forme de deux porteuses en quadratures modulées en amplitude par $\cos \left(\phi_{k}\right) \sin \left(\phi_{k}\right)$

et $\quad$ Ceci illustre la similitude entre la modulation de phase et la modulation d'amplitude qui peuvent être combinées pour obtenir la Modulation d'Amplitude en Quadrature (MAQ). 


\section{Constellation d'une modulations PSK-4}

\begin{tabular}{|cc:l|}
\hline bit 1 & bit & $\phi_{\mathrm{k}}$ \\
\hline 0 & 0 & $\pi / 4$ \\
0 & 1 & $3 \pi / 4$ \\
1 & 1 & $-3 \pi / 4$ \\
1 & 0 & $-\pi / 4$ \\
\hline
\end{tabular}

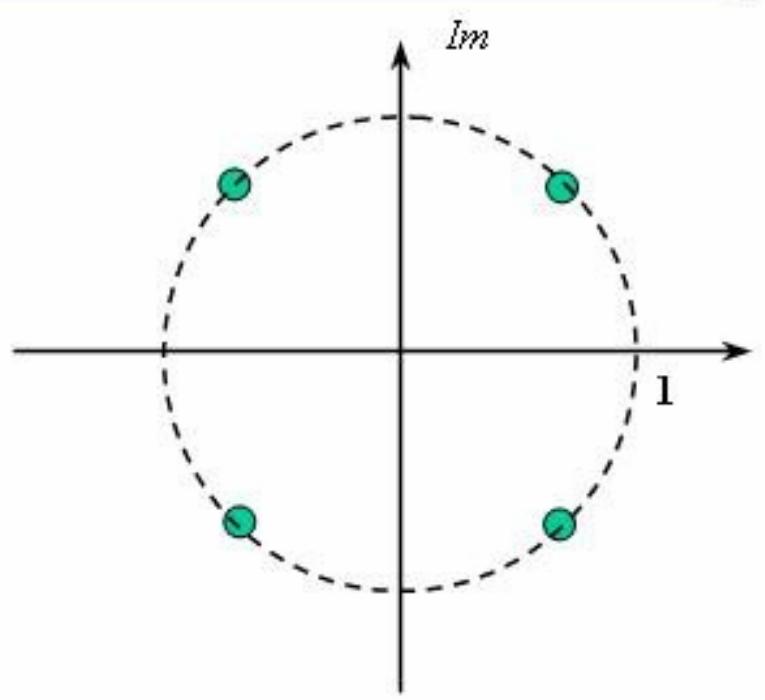

Fig. 4. Modulation PSK-4 : constellation et dictionnaire associé.

\subsection{Modulation d'Amplitude en Quadrature (MAQ)}

La modulation d'amplitude en quadrature s'écrit :

$$
\begin{gathered}
x(t)=u_{c}(t) \cos \left(\omega_{0} t\right)-u_{s}(t) \sin \left(\omega_{0} t\right) \\
\text { avec } u_{c}(t)=\sum_{k} a_{k} h(t-k T) \text { d } u_{s}(t)=\sum_{k} b_{k} h(t-k T)
\end{gathered}
$$

Le signal $x(t)$ est donc la somme de deux porteuses en quadrature, modulées en amplitude par les signaux $u_{c}(t)$ et $u_{s}(t)$. Les $a_{k}$ et $b_{k}$ représentent des symboles $M$-aires qui prennent leurs valeurs dans un alphabet à $M$ éléments. Ainsi on obtient une modulation à $\mathrm{M}^{2}$ états, chacun étant associé à un couple de symbole M-aires. La figure 5 montre les constellations des modulations MAQ-16 avec son dictionnaire associé. 


\section{Constellation d'une modulations MAQ-16}

\begin{tabular}{|cc:c|cc:c|}
\hline bit 1 bit 2 & $\mathbf{a}_{\mathbf{k}}$ & bit 3 bit 4 & $\mathbf{b}_{\mathbf{k}}$ \\
\hline 0 & 0 & +3 & 0 & 0 & +3 \\
0 & 1 & +1 & 0 & 1 & +1 \\
1 & 1 & -1 & 1 & 1 & -1 \\
1 & 0 & -3 & 1 & 0 & -3 \\
\hline
\end{tabular}

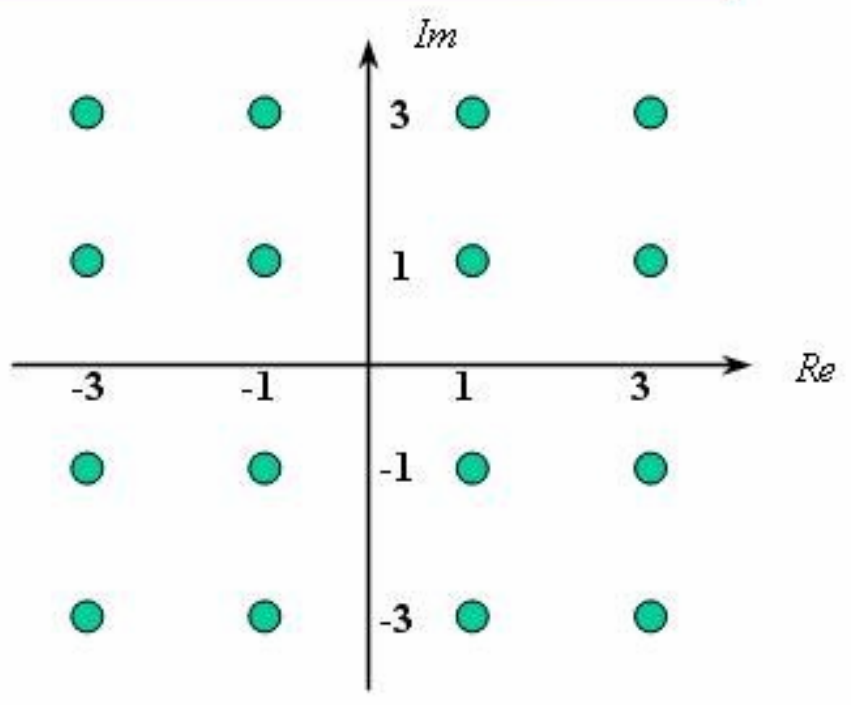

Fig. 5. Modulation MAQ-16 : constellation et dictionnaire associé.

Les schémas classiques des récepteurs numériques contiennent une partie dédiée à la démodulation synchrone. L'application de cette technique suppose la mise en oeuvre d'un système de récupération de porteuse dont l'élément central est la boucle à verrouillage de phase. Nous proposons dans le chapitre suivant d'aborder ce problème par une étude de la stabilité de la boucle à verrouillage de phase.

\section{Démodulation numérique synchrone}

Le propos de cet article n'est pas de passer en revue les différentes structures des récepteurs numériques en fonction du type de modulation utilisé. C'est pourquoi nous illustrons le problème de la détection synchrone à partir d'une modulation de phase PSK dont le récepteur optimal est représenté sur la figure 6. La première étape consiste à transposer le signal reçu en bande de base, c'est-à-dire de retrouver l'information telle qu'elle était avant l'opération de modulation, suivant la technique classique de détection synchrone. Lors de la seconde étape le signal est filtré pour ne conserver que le résultat de cette transposition ; enfin il est échantillonné, puis transmis à un organe de décision dont la forme la plus simple est un comparateur.

Dans le paragraphe suivant, nous allons étudier le comportement d'une PLL (Phase Lock Loop) qui est utilisée dans le système de récupération de porteuse. 


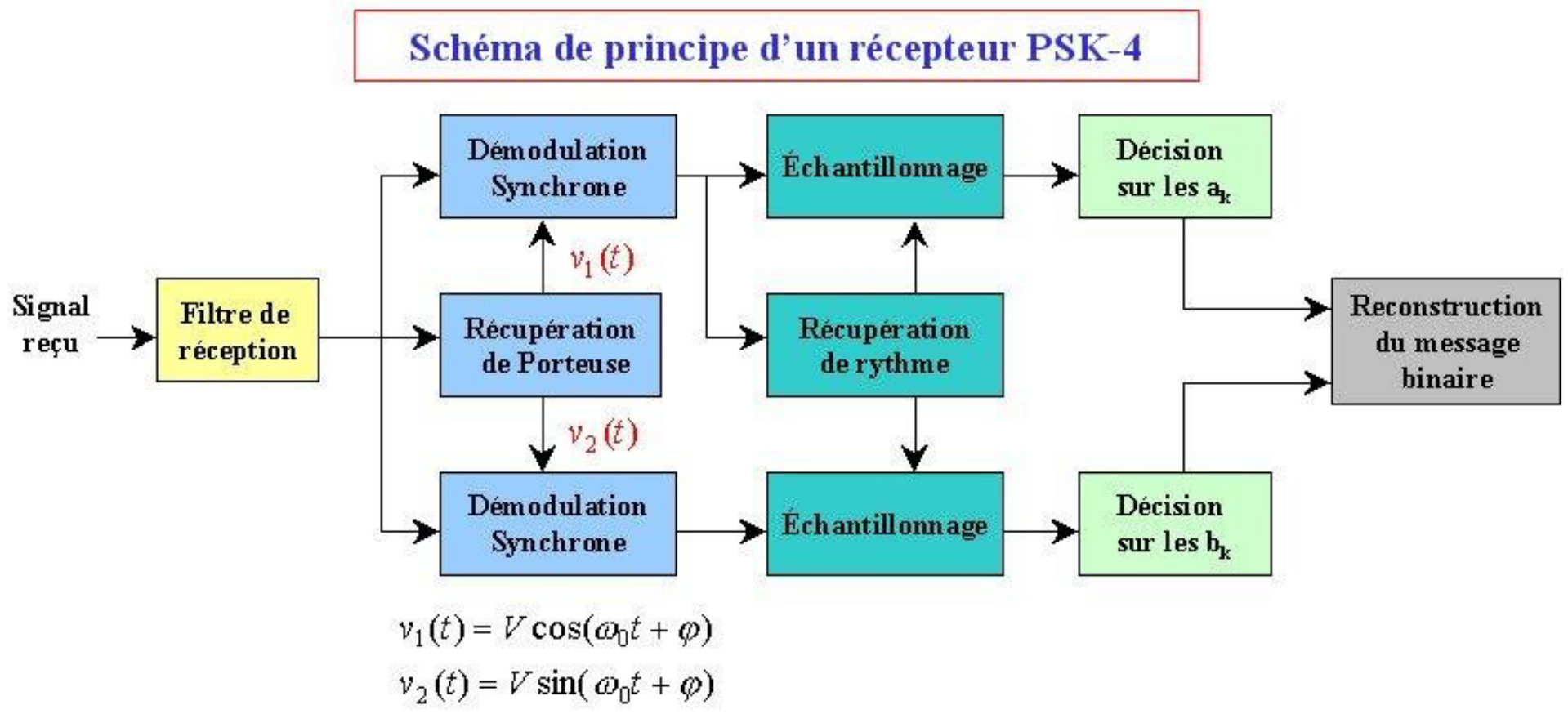

Fig. 6. Schéma bloc d'un récepteur numérique.

Filtre de réception : en général un filtre passe-bande accordé autour de la fréquence porteuse. Il permet d'une part de sélectionner le signal à traiter et d'autre part de diminuer la puissance du bruit.

Démodulation synchrone : il s'agit d'une part de multiplier le signal modulé reçu par un signal de même fréquence que la porteuse, et d'autre part de filtrer (filtre passa-bas) le signal résultant pour en extraire le signal modulant.

Récupération de porteuse : dispositif qui permet de générer un signal de référence de même fréquence et de phase connue par rapport à la porteuse de la modulation (PLL, boucle de Costas ...).

Echantillonnage : piloté par le dispositif de récupération de rythme, il assure un échantillonnage synchrone des symboles transmis.

Récupération de rythme : dispositif qui permet de générer un signal synchrone avec le rythme d'émission du message numérique. Il est élaboré à partir du message numérique démodulé.

Décision : dispositif permettant de quantifier les symboles échantillonnés et de leur donner leur valeur initiale lors de l'émission. En général il s'agit d'un simple comparateur à seuil.

\subsection{Stabilité de la PLL}

Le schéma de principe d'une PLL est donné en figure 7, il est composé d'un comparateur de phase dont le signal $\varepsilon(t)$

de sortie est , d'un filtre de boucle dont la fonction de transfert est supposée être celle d'un filtre passe-bas et d'un oscillateur contrôlé en tension (OCT) dont le signal de sortie sera noté v(t) :

$$
v(t)=V_{0} \cos \left(\omega_{0} t+\varphi\right)
$$




\section{Schéma de principe d'une PLL}

entrée

$u(t)$

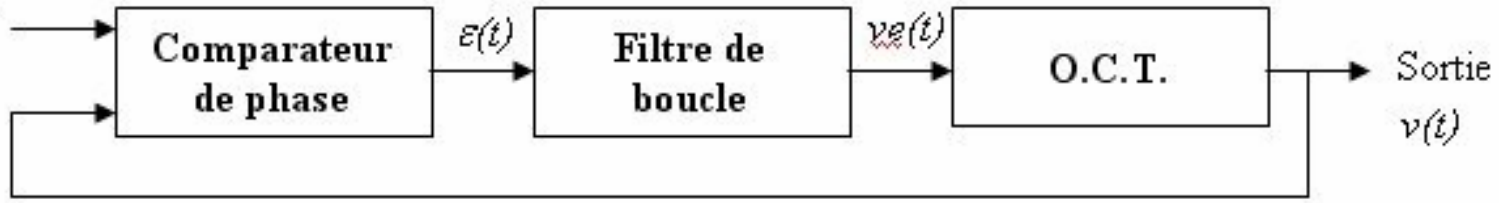

Fig. 7. Schéma de Principe d'une PLL.

Les équations simplifiées correspondantes sont respectivement pour le comparateur et pour l'OCT :

$$
\varepsilon(t)=K u(t) v(t) \text { et } \frac{d \varphi}{d t}=K_{0} v_{e}(t)
$$

Nous allons étudier le comportement de la PLL du point de vue de sa stabilité, lorsque le signal d'entrée u(t) est analogique et modulé en amplitude de la forme :

$$
u(t)=U_{0}(1+m g(t)) \cos \left(\omega_{0} t\right)
$$

$$
|g(t)| \leq 1
$$

Le signal modulant $\mathrm{g}(\mathrm{t})$ est considéré à moyenne nulle et tel que de module maximum inférieur à 1 , ainsi on peut distinguer les deux cas classiques de modulation d'amplitude, représentées sur la figure $8:$ la modulation d'amplitude avec porteuse pour laquelle $\mathrm{m}<1$ et avec résidu de porteuse pour laquelle $\mathrm{m}>1$.

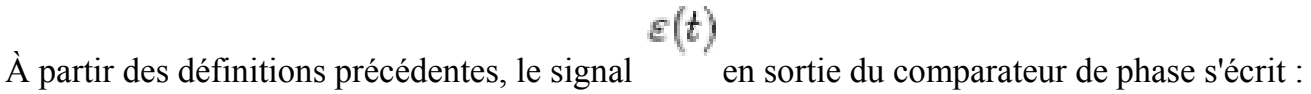

$$
\varepsilon(t)=K U_{0} V_{0}[1+m g(t)]\left[\cos \left(2 \omega_{0} t+\varphi\right)+\cos \varphi\right]
$$

$2 \omega_{0}$

le terme en disparaît après le filtrage passe-bas et si on considère que le gain statique du filtre est égal à 1 alors on peut écrire :

$$
\frac{d \varphi}{d t}=\frac{K K_{0} U_{0} V_{0}}{2}[1+m g(t)] \cos \varphi
$$

sous réserve que :

$$
\left|\frac{d \varphi}{d t}\right|<\omega_{c}
$$

\footnotetext{
où ${ }_{c}{ }_{c}$ représente la pulsation de coupure du filtre passe-bas.
}

En régime permanent établi on a donc

$$
d \varphi / d t=0 \quad \varphi_{A}=+\pi / 2
$$

$$
\varphi_{B}=-\pi / 2 \text { obtenus à partir de } \cos \varphi=0
$$

et

$\varphi(t)$ 
étant difficilement exploitable, on se contente d'étudier le comportement asymptotique de vers l'infini.

Modulation $\mathbf{A M}$ avec $\mathbf{m}<1$

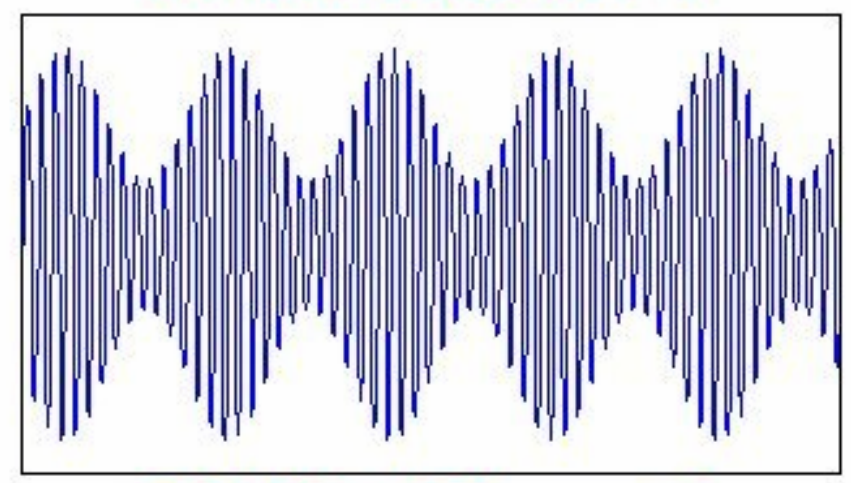

Modulation $A M$ avec $m>1$

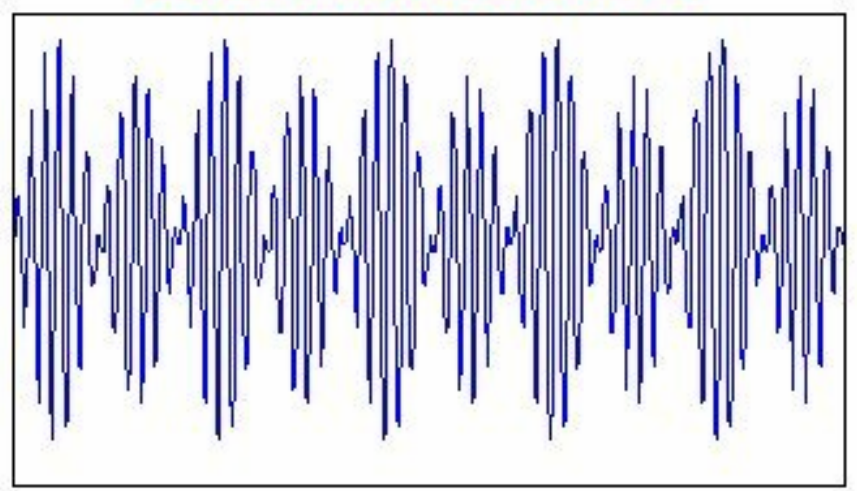

$\mathrm{U}(\mathrm{t})=\mathrm{U}_{0}(1+\mathrm{mg}(\mathrm{t})) \cos \left(\omega_{0} \mathrm{t}\right)$

Fig. 8. Modulation d'amplitude avec porteuse $\mathrm{m}<1$ et avec résidu de porteuse $\mathrm{m}>1$.

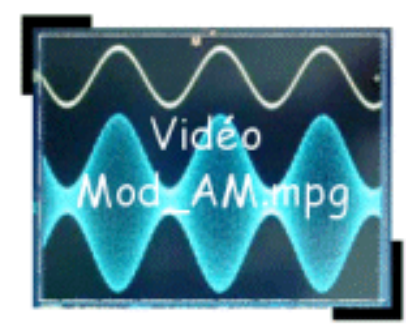

Vidéo 1. Modulation d'amplitude analogique : variation de l'indice de modulation (1,6 Mo, format MPEG)

\subsubsection{Cas d'une modulation d'amplitude avec porteuse}

L'étude de la stabilité autour du point A est conduite en posant :

$$
\varphi=\varphi_{A}+\theta=\frac{\pi}{2}+\theta
$$

$\theta$ correspondant à un écart autour du point $\mathrm{A}$ au temps $\mathrm{t}^{\prime}=0$, l'objectif est de calculer la valeur ${ }^{\theta_{\infty}}$ prise par $\theta$ quand $t$ tend vers l'infini en intégrant l'équation suivante : 


$$
\frac{d \theta}{d t}=-C[1+m g(t)] \sin \theta
$$

le déroulement du calcul est proposé en annexe A, nous ne présentons ici que les résultats obtenus. La solution

finale est donc donnée par $\theta_{\infty}=2 k \pi$ ou bien $\varphi_{\infty}=\pi / 2$. Il y a donc équilibre stable autour du point $\varphi_{A}=\pi / 2$

. Par un raisonnement analogue, l'équilibre au point B est étudié en posant :

On trouve alors comme résultat $\theta_{\infty}=\bar{\pi}+2 k \bar{\pi}$ et donc que $\varphi_{A} \equiv \pi / 2$. La conclusion de ce calcul est

$$
\varphi=\varphi_{B}+\theta=-\frac{\pi}{2}+\theta
$$

alors que le point A est le seul point stable de la PLL. En résumé, après un régime transitoire, la phase ${ }^{\text {de }}$ $+\pi / 2$

l'OCT se fixe à la valeur par rapport au signal d'entrée $u(t)$ indépendamment des ses variations d'amplitude.

\subsubsection{Cas d'une modulation d'amplitude avec $m>1$}

Le calcul s'effectue de la même façon que dans le cas précédent, il est décrit en annexe B. Dans le cas d'une modulation sans porteuse ( $m=\infty$ ), avec signal modulant sinusoïdal de pulsation $\Omega$, l'entrée s'écrit :

$$
u(t)=U_{0} \cos (\Omega t) \cos \left(\omega_{0} t\right)
$$

on montre alors qu'il n'existe pas de solution asymptotique stable ${ }^{\theta_{\infty}}$. Dans ce cas, il est fait appel à une double boucle à verrouillage de phase ou boucle de Costas.

\subsubsection{La boucle de Costas}

Le schéma de principe est représenté figure 9. Les blocs M1, M2 et M3 sont des multiplieurs jouant le rôle de comparateurs de phase. 


\section{Principe de la boucle de Costas}

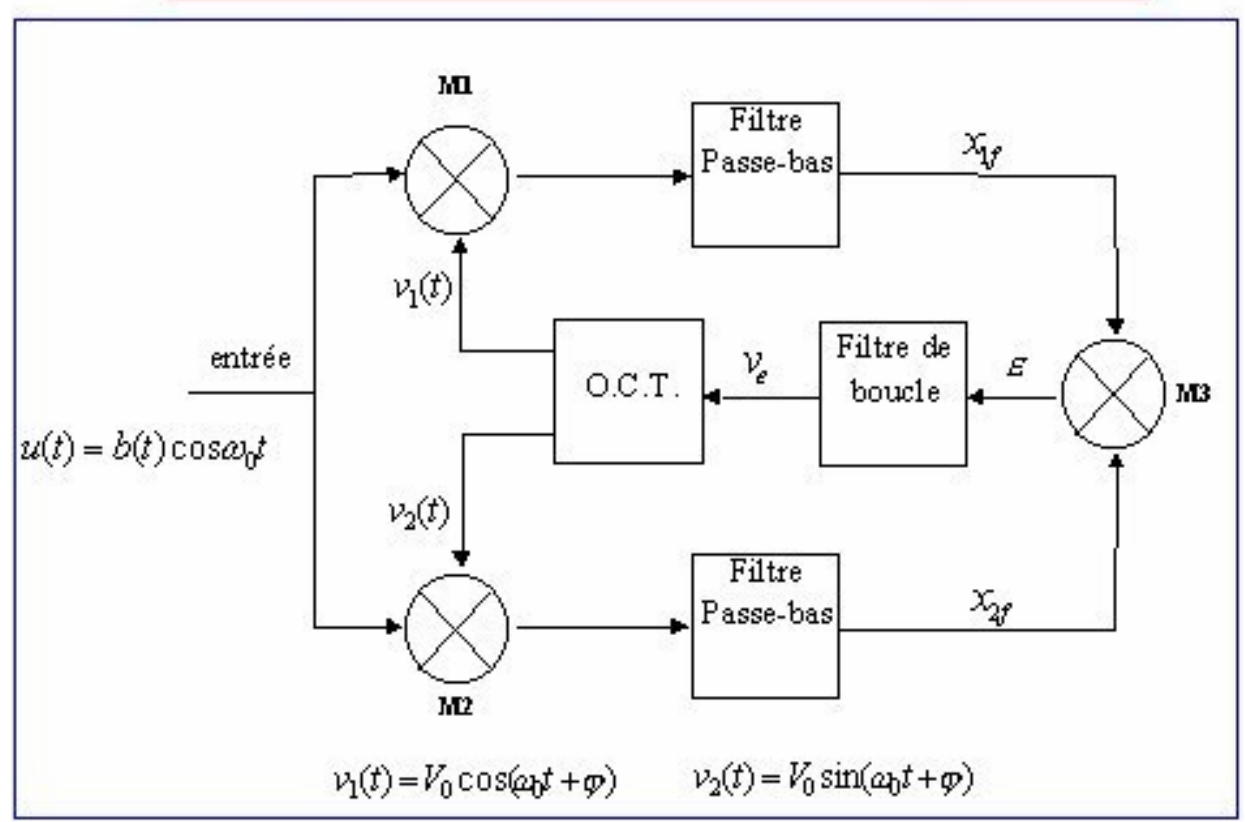

Fig. 9. Schéma bloc d'une boucle de Costas.

Soit $u(t)=U_{0 g}(t) \cos \left(\omega_{0} t\right)$

obtient l'équation différentielle suivante :

$$
\begin{array}{r}
\frac{d \varphi}{d t}=K_{0} \frac{U_{0}^{2} V_{0}^{2}}{8}[g(t)]^{2} \sin (2 \varphi) \\
\sin (2 \varphi)=0
\end{array}
$$

qui montre l'existence de 4 points d'équilibre obtenus pour

. En utilisant le changement de $\varphi=\varphi_{\text {equilibre }}+\theta$

points d'équilibre stables $A_{1}$ et $A_{2}$ de valeurs $\varphi_{\infty}= \pm \pi / 2$. Dans ces conditions, les deux sorties de l'OCT s'écrivent :

$$
v_{1}(t)=\mp V_{0} \sin \left(\omega_{0} t\right) \quad v_{2}(t)= \pm V_{0} \cos \left(\omega_{0} t\right)
$$

Ces deux signaux en quadrature sont à phase stable, leur signe dépendant du point d'équilibre sur lequel s'est fixé la boucle à la mise sous tension. Le signal démodulé est extrait directement en $\mathrm{x}_{2 \mathrm{f}}$, selon un schéma classique de détection synchrone :

$$
\begin{gathered}
x_{1 f}=\frac{U_{0}^{2} V_{0}^{2}}{8} g(t) \cos \varphi=0 \\
x_{2 f}=\frac{U_{0}^{2} V_{0}^{2}}{8} g(t) \sin \varphi= \pm \frac{U_{0}^{2} V_{0}^{2}}{8} g(t)
\end{gathered}
$$




\section{Réalisation pédagogique}

\subsection{La maquette d'étude}

Pour illustrer les problèmes précédents, nous avons construit une maquette permettant la réalisation de diverses fonctions de modulation et démodulation d'amplitude ainsi que la réalisation de boucles à verrouillage de phase. Cette maquette, représentée sur la figure 10, comprend 4 multiplieurs analogiques M1 à M4, type AD 633, et un OCT à 2 sorties en quadrature (type 8038 et diviseur associé à 4013).

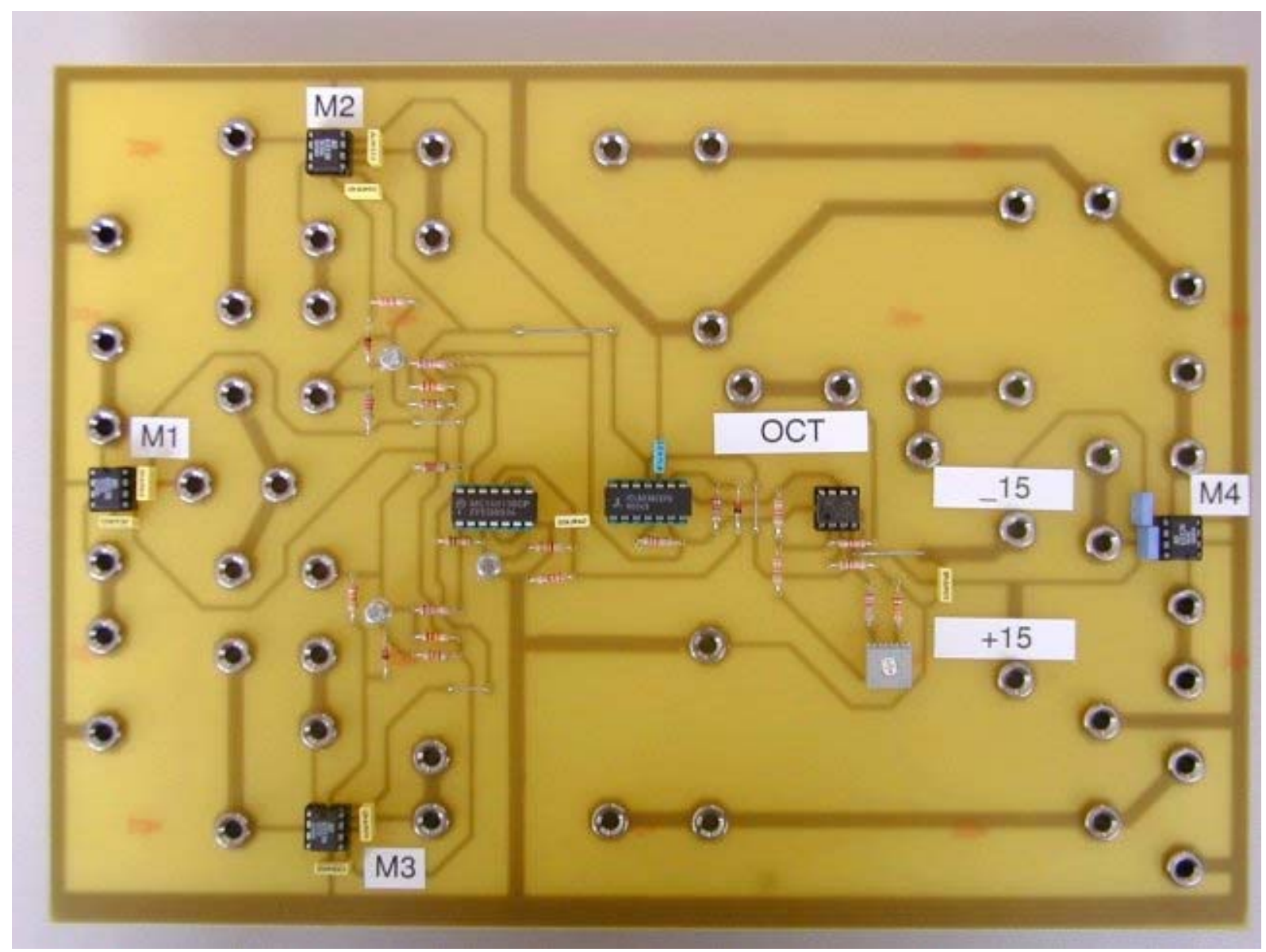

Fig. 10. Présentation de la maquette d'étude.

Les signaux modulés en amplitude sont générés par le multiplieur M1, au moyen de deux générateurs basse fréquence, l'un pour la porteuse à $\mathrm{f}_{0}$ et l'autre pour le signal modulant $\mathrm{g}(\mathrm{t})$. Ce dernier générateur comprend en outre un réglage d'offset qui permet d'obtenir un taux de modulation $\mathrm{m}$ variable de 0 à l'infini. Par exemple, une modulation PSK-2 pourra être réalisée en prenant pour $\mathrm{g}(\mathrm{t})$ un signal carré à moyenne nulle. Les symboles binaires associés sont :

e qui correspond au codage en ligne NRZ.

$$
a_{k}=1 \quad \text { si } g(t)>0 \quad \text { et } \quad a_{k}=-1 \quad \text { si } g(t)<0
$$

Le schéma bloc de la maquette est représenté figure 11, les schémas électroniques des différents parties : l'OCT à deux sorties en quadratures et le circuit de mise en forme sont représentés respectivement sur les figures 12-a, 12 -b et 13 . 


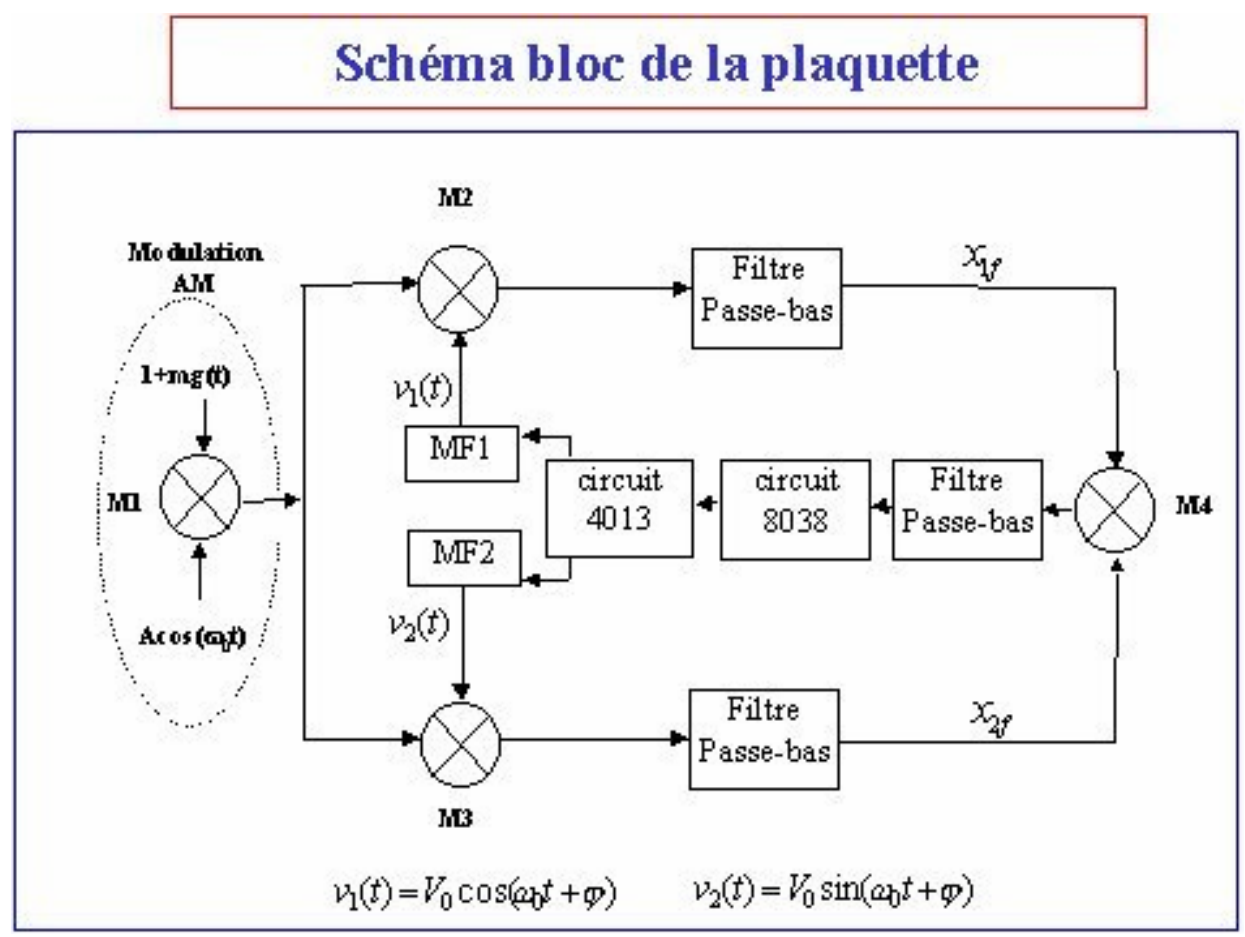

Fig. 11. Schéma bloc de la maquette.

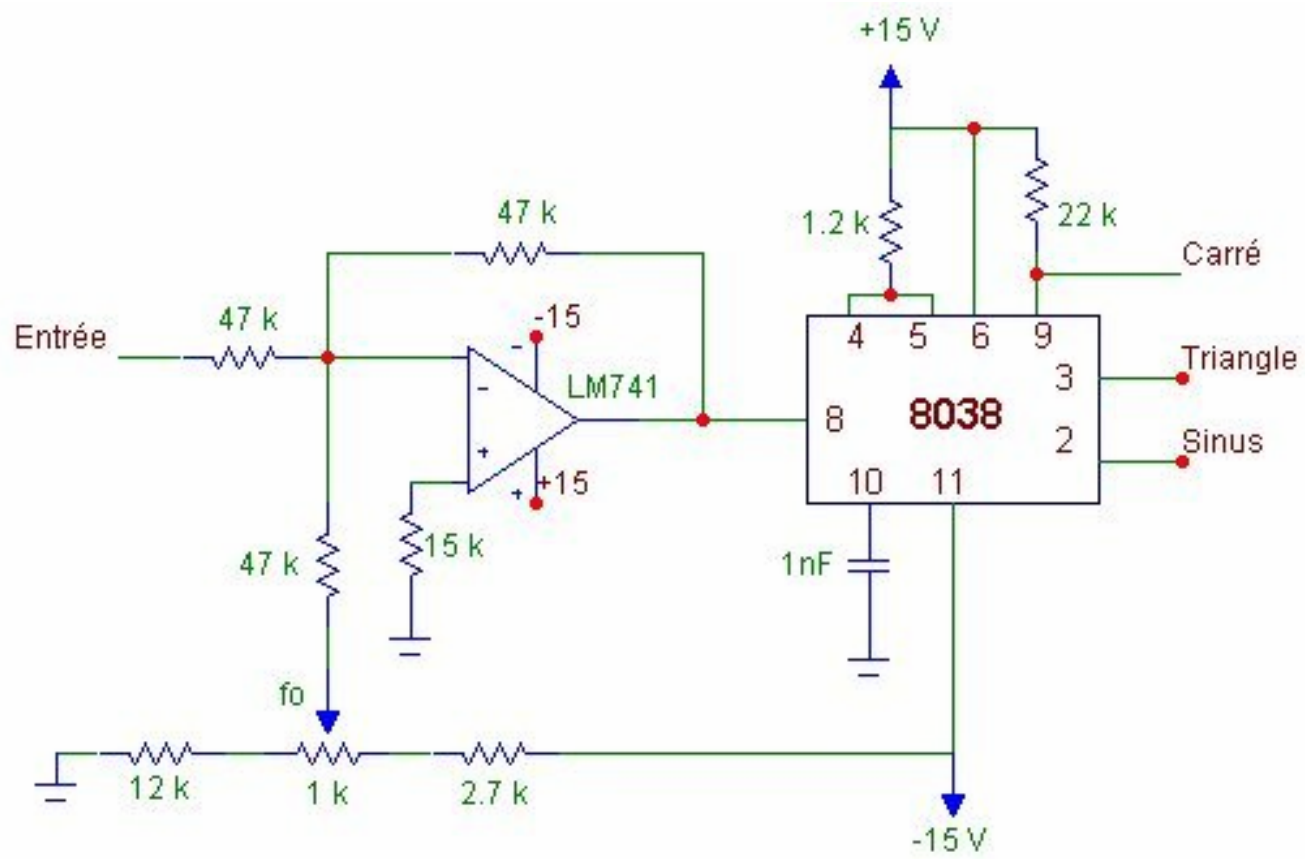




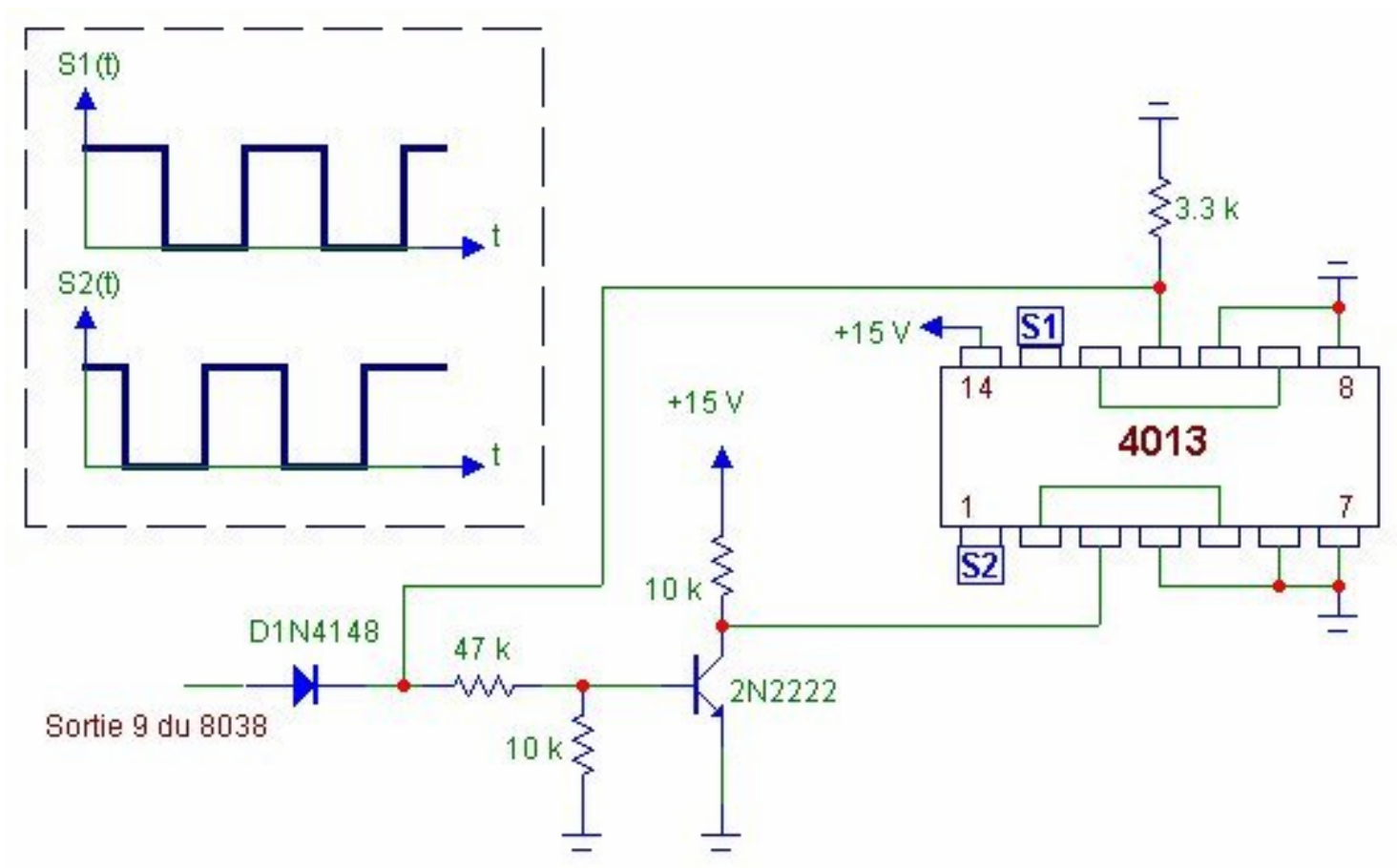

Fig. 12. Schéma de l'OCT à deux sorties en quadratures. Haut) Oscillateur contrôlé en tension. Bas) Circuit de génération de signaux en quadratures.

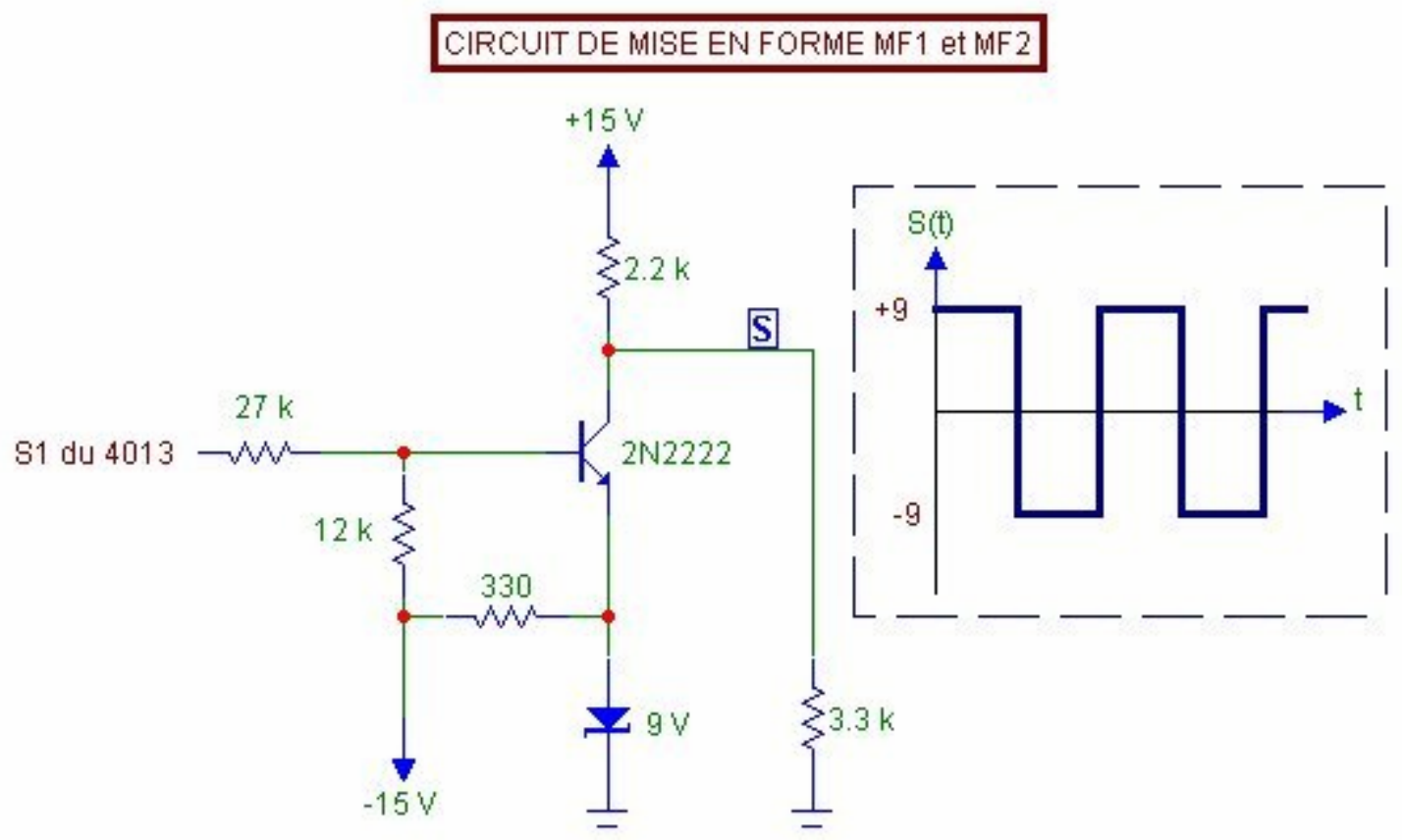

Fig. 13. Schéma du circuit de mise en forme. 


\subsection{Quelques résultats}

Les figures 14 et 15 montrent les signaux obtenus lors du fonctionnement en démodulation d'amplitude avec et sans porteuse, les figures 16 et 17 illustrent la récupération de porteuse et la démodulation d'un signal numériques PSK-2.

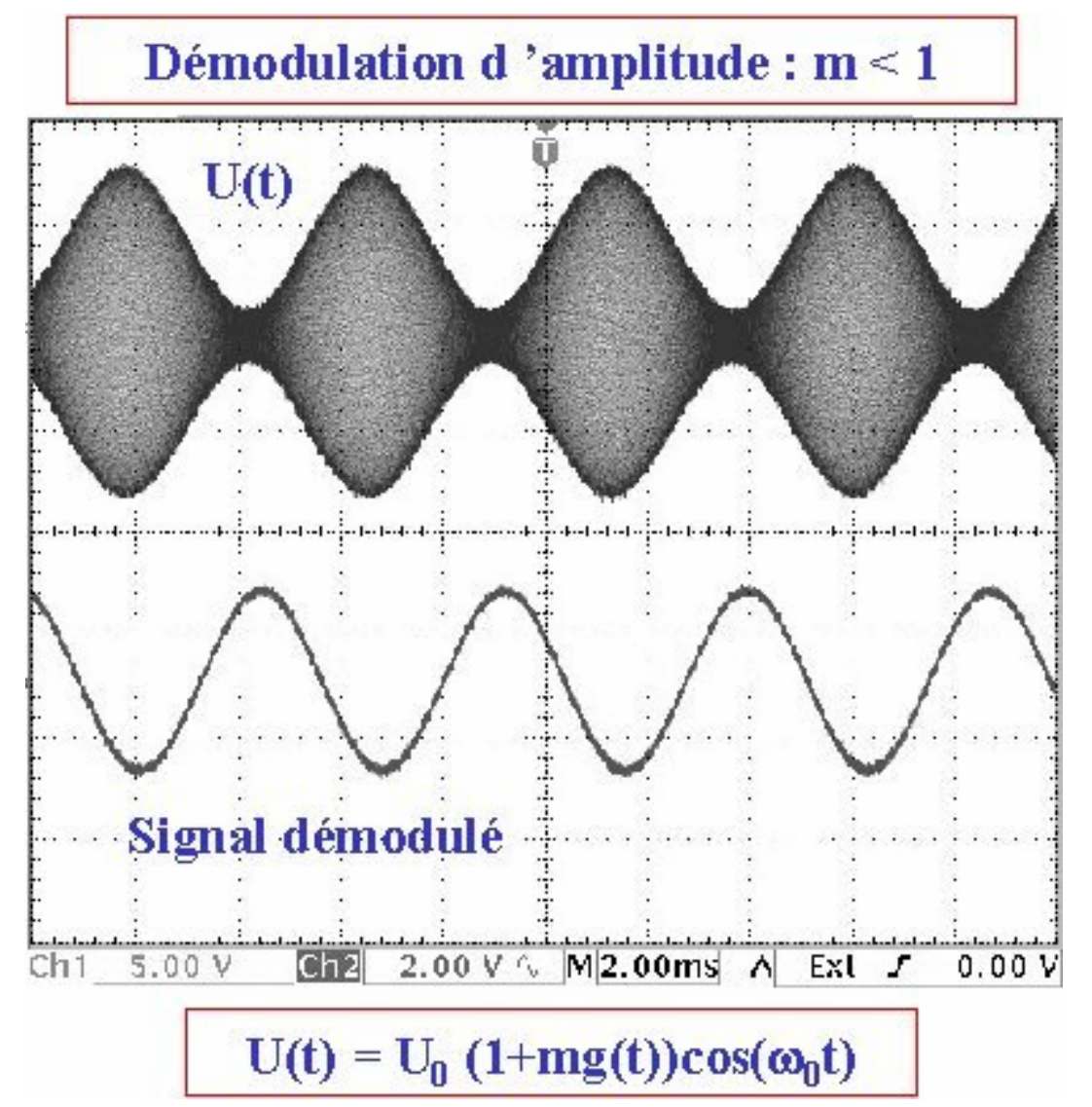

Fig. 14. Démodulation d'amplitude avec porteuse. 


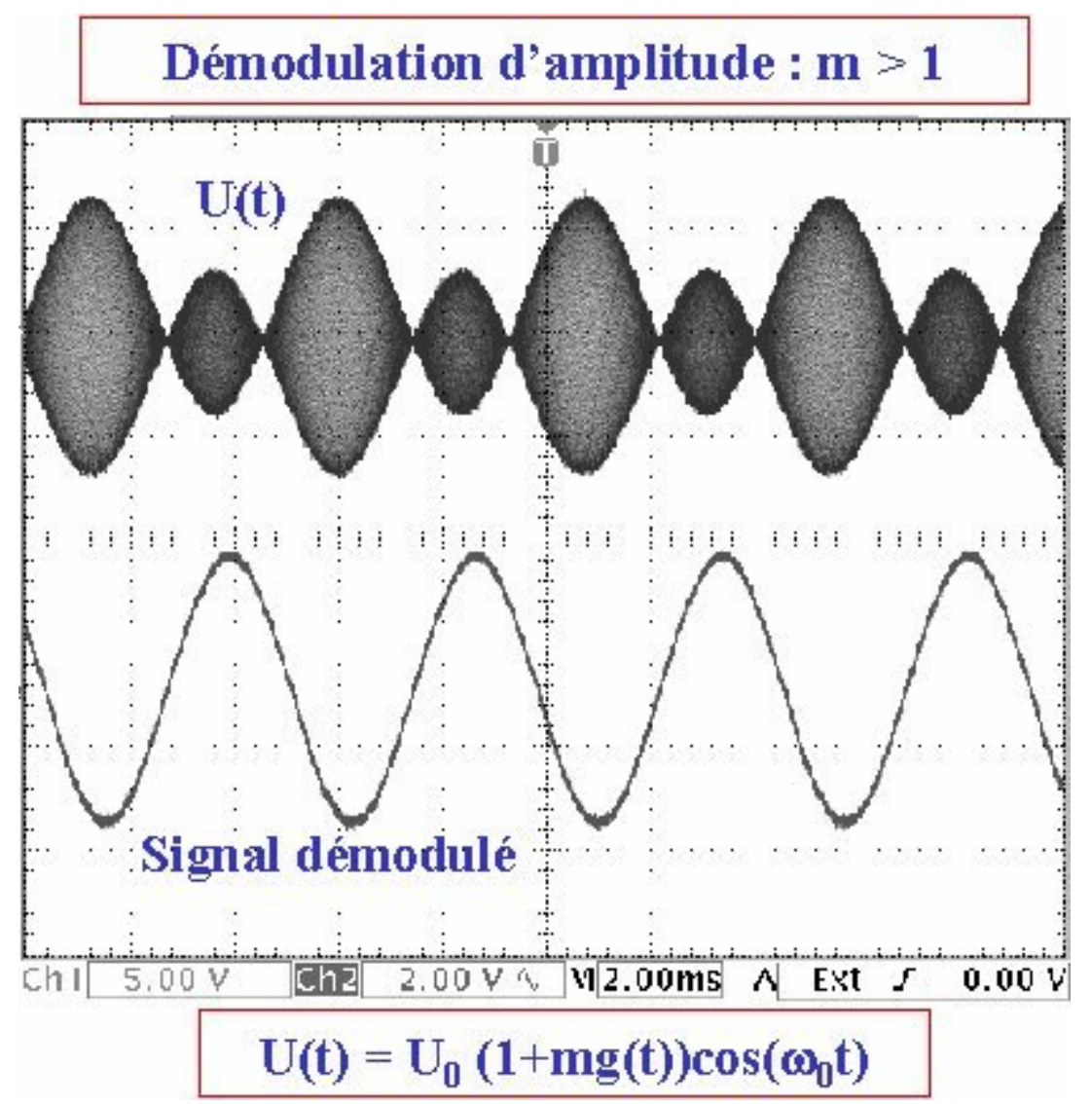

Fig. 15. Démodulation d'amplitude avec résidu de porteuse.

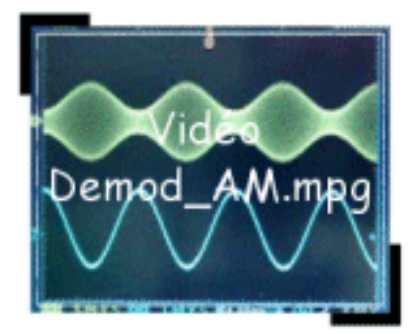

Vidéo 2. Démodulation d'amplitude par boucle de Costas (2,1 Mo, format MPEG) 


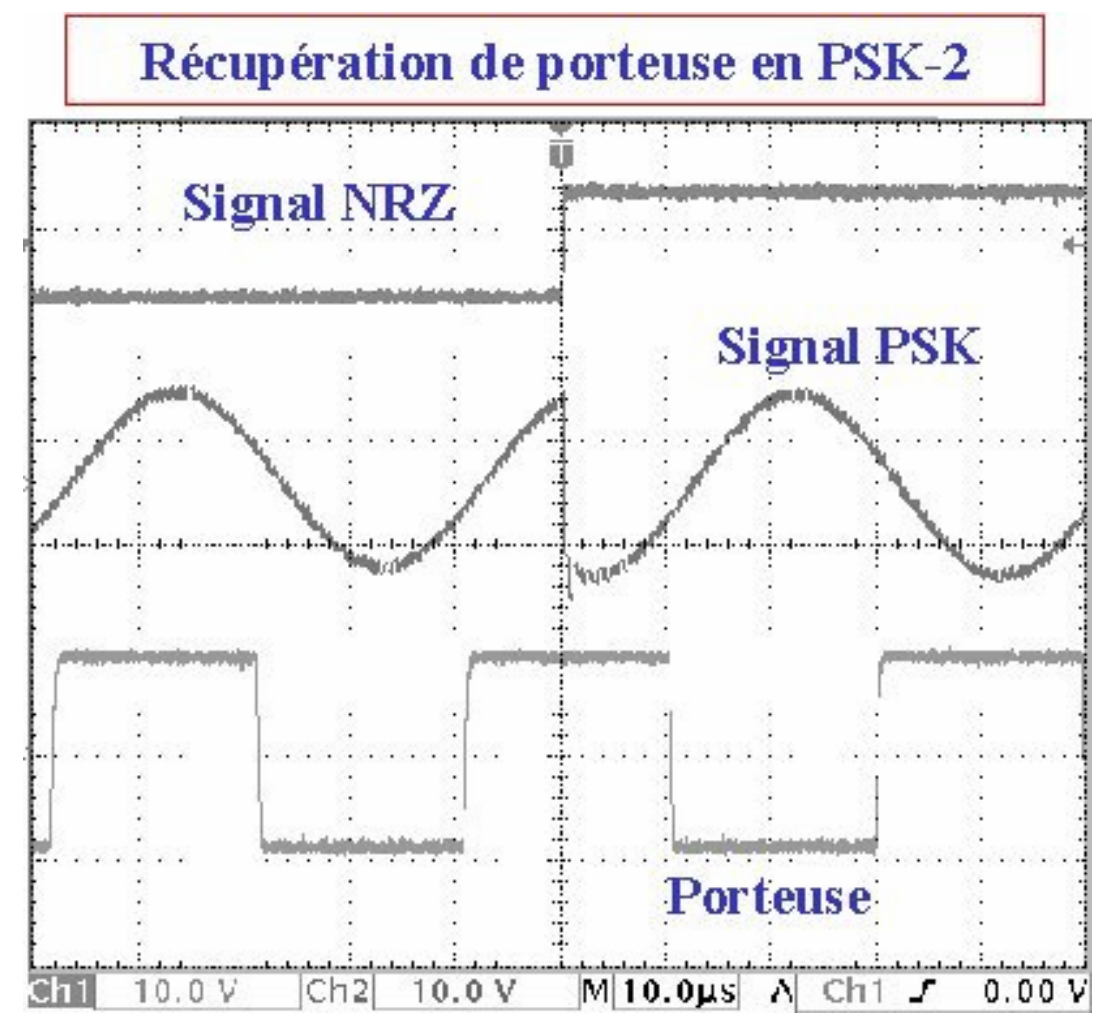

Fig. 16. Récupération de porteuse en PSK-2, la porteuse est obtenue en sortie du circuit de mise en forme (Fig. 13).

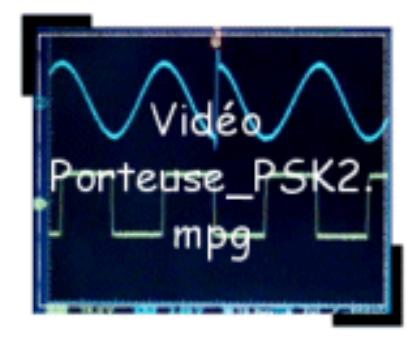

Vidéo 3. Modulation PSK-2 non cohérente (2,0 Mo, format MPEG) 


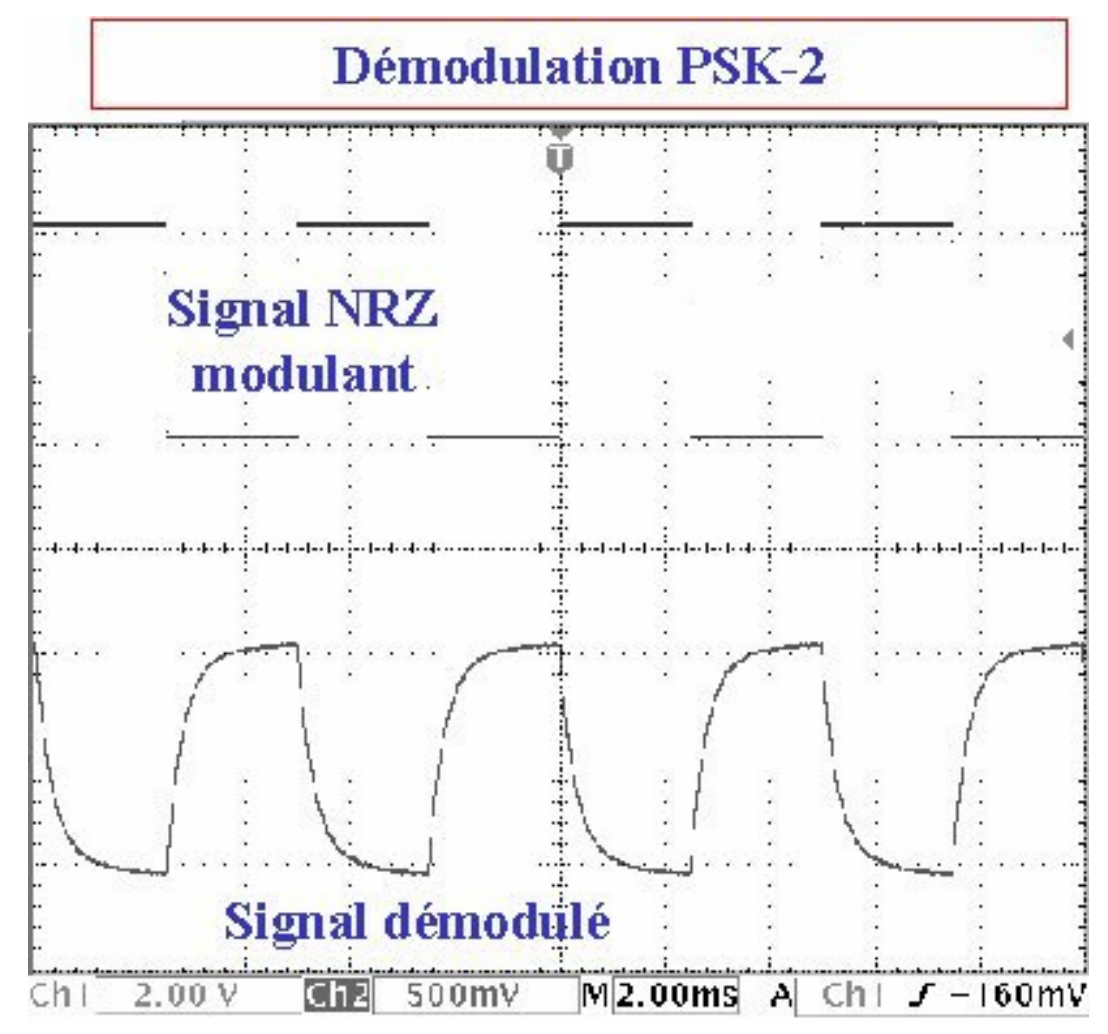

Fig. 17. Démodulation d'un signal PSK-2.

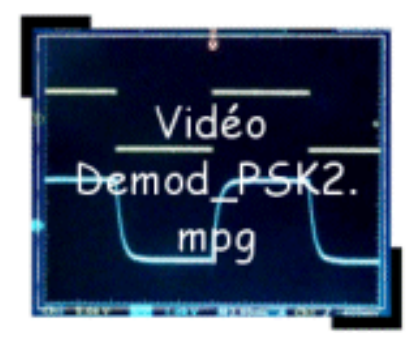

Vidéo 4. Démodulation PSK-2 par boucle de Costas (2,1 Mo, format MPEG)

\section{Conclusion}

Cette maquette a été utilisée dans le cadre d'un stage inscrit au Plan Académique de Formation de l'académie de Poitiers en 2001, à destination de professeurs STI du second degré des lycées techniques. Elle a permis de sensibiliser le public concerné à la stabilité des boucles à verrouillage de phase rencontrées dans les systèmes de réception analogiques et numériques. Elle est actuellement exploitée dans des enseignements de licence ou maîtrise EEA. La réalisation du TP sur la boucle de Costas nécessite comme pré-requis une bonne connaissance du comportement de la boucle à verrouillage de phase.

\section{Remerciements}

Les auteurs remercient Jean-Jacques Villessange, technicien du département EEA, pour son aide précieuse sur la réalisation pratique de la carte. 


\section{Bibliographie}

J. Encinas, Systèmes à verrouillage de phase (Masson, 1990).

M. Girard, Boucle à verouillage de phase (Mc Graw Hill, 1991).

A. Glavieux et M. Joindot, Communications numériques, introduction (Masson, 1996).

J.C. Bic, D. Duponteil et J.C. Imbeaux, Éléments de communications numériques, tome 1 et 2 (Dunod, 1986).

\section{Annexe A. Stabilité d'une PLL pour un signal d'entrée modulé AM avec porteuse}

Le signal modulant $\mathrm{g}(\mathrm{t})$ est supposé à moyenne nulle, et l'indice de modulation $\mathrm{m}$ est inférieur à 1 . L'étude de la stabilité autour du point A est conduite en posant :

$$
\varphi=\varphi_{A}+\theta=\frac{\pi}{2}+\theta
$$

En reprenant l'équation suivante décrite dans l'article :

$$
\frac{d \varphi}{d t}=\frac{K K_{g} U_{0} V_{0}}{2}[1+m g(t)] \cos \varphi
$$

qui devient alors :

$$
\frac{d \theta}{d t}=-C[1+m g(t)] \sin \theta
$$

dans laquelle $\mathrm{C}$ est une constante positive :

$$
C=\frac{K K_{0} U_{0} V_{0}}{2}
$$

Pour trouver une solution à $\theta$, il faut intégrer l'équation (3) en utilisant l'expression :

$$
\int \frac{d x}{\sin x}=\ln \left|\tan \frac{x}{2}\right|
$$

L'intégrale prend alors la forme suivante :

$$
\begin{gathered}
\frac{d \theta}{\sin \theta}=-C[1+m g(t)] d t \\
\int_{\theta_{0}}^{\theta} \frac{d x}{\sin x}=\left[\ln \left|\tan \frac{x}{2}\right|\right]_{\theta_{0}}^{\theta}=-C \int_{0}^{t}[1+m g(\tau)] d \tau
\end{gathered}
$$

Soit :

$$
\varliminf_{n}\left|\tan \frac{\theta}{2}\right|=\ln \left|\tan \frac{\theta_{a}}{2}\right|-I(t)
$$

avec 


$$
\begin{gathered}
I(t)=-C \int_{0}^{t}[1+m g(\tau)] d \tau \\
\left|\tan \frac{\theta}{2}\right|=\left|\tan \frac{\theta_{0}}{2}\right| \cdot \exp (-I(t))
\end{gathered}
$$

$$
\begin{aligned}
& I(t) \underset{t \rightarrow \infty}{\longrightarrow} \infty \quad \exp (-I(t)) \rightarrow 0 \\
& \text { puisque } \mathrm{m}<1 \text {, alors }(1+\mathrm{mg}(\mathrm{t}))>0 \text { et nous avons } \\
& \text { et } \text {. Sous réserve que } \\
& \theta_{0} \neq k \pi \text {, il s'ensuit que }\left|\tan \frac{\theta}{2}\right| \underset{t \rightarrow \infty}{\longrightarrow} \text {. La solution finale est donc donnée par } \theta_{\infty}=2 k \pi \text { ou bien } \\
& \varphi_{\infty}=\pi / 2 \text {. Il y a équilibre stable au point } \varphi_{A}=\pi / 2
\end{aligned}
$$

Par un raisonnement analogue, l'équilibre au point B est étudié en posant :

$$
\varphi=\varphi_{B}+\theta=-\frac{\pi}{2}+\theta
$$

l'équation (3) devient :

$$
\frac{d \theta}{d t}=-C[1+m g(t)] \sin \theta
$$

donc

$$
I(t)=-C \int_{0}^{t}[1+m g(\tau)] d \tau<0
$$

alors

$$
\exp (-I(t)) \underset{t \rightarrow \infty}{\longrightarrow}+\infty
$$

l'équation (9) ne peut être satisfaite que si

$$
\left|\tan \frac{\theta}{2}\right| \rightarrow+\infty
$$

soit

$$
\frac{\theta_{\mathrm{o}}}{2}=\frac{\pi}{2}+k \pi
$$

On trouve alors que

$$
\theta_{\infty}=\pi+2 k \pi
$$

et

$$
\varphi_{\infty}=-\frac{\pi}{2}+\theta_{\infty}=+\frac{\pi}{2}
$$


ce qui correspond au point $\mathrm{A}$.

En résumé, après un régime transitoire dont la durée dépend du terme $\exp (-I(t))^{\varphi}$, la phase ${ }^{\varphi}$ d'OCT se fixe à la valeur ${ }^{+\pi / 2}$, indépendamment des variations d'amplitude de l'entrée $u(t)$.

\section{Annexe B. Stabilité d'une PLL pour un signal d'entrée modulé AM sans porteuse}

La valeur de la solution asymptotique de l'équation (9) dépend du comportement de l'intégrale I(t). Si le terme $\exp (-I(t))$ ne tend pas vers zéro au cours du temps, on ne peut pas garantir la stabilité du point A. Par exemple, dans le cas d'une modulation sans porteuse, avec signal modulant sinusoïdal de pulsation $\Omega$, l'entrée s'écrit :

$$
u(t)=U_{0} \cos (\Omega t) \cos \left(\omega_{0} t\right)
$$

et l'intégrale $\mathrm{I}(\mathrm{t})$ devient :

$$
I(t)=C \int_{0}^{t} \cos (\Omega \tau) d \tau=\frac{C}{\Omega} \sin (\Omega t)
$$

Il n'existe alors pas de solution asymptotique stable ${ }^{\infty}$ de l'équation (9). Dans ce cas, il est fait appel à une double boucle à verrouillage de phase ou boucle de Costas.

\section{Annexe C. Exemple d'un texte de TP : Récupération de porteuse et démodulation}

On dispose d'une maquette contenant les fonctions suivantes :

- 1 OCT à 2 sorties en quadrature à 8038 ;

- 4 multiplieurs analogiques AD633.

Cette maquette permet donc la réalisation de diverses fonctions de modulation et démodulation d'amplitude ainsi que la réalisation de boucles à verrouillage de phase. Les signaux modulés en amplitude sont tous générés par le multiplieur M1 situé sur la partie gauche de la maquette, au moyen de 2 générateurs basse fréquence, l'un pour la porteuse à $\mathrm{f}_{0}$ et l'autre pour le signal modulant $\mathrm{b}(\mathrm{t})$, selon le montage décrit en figure 18 . 


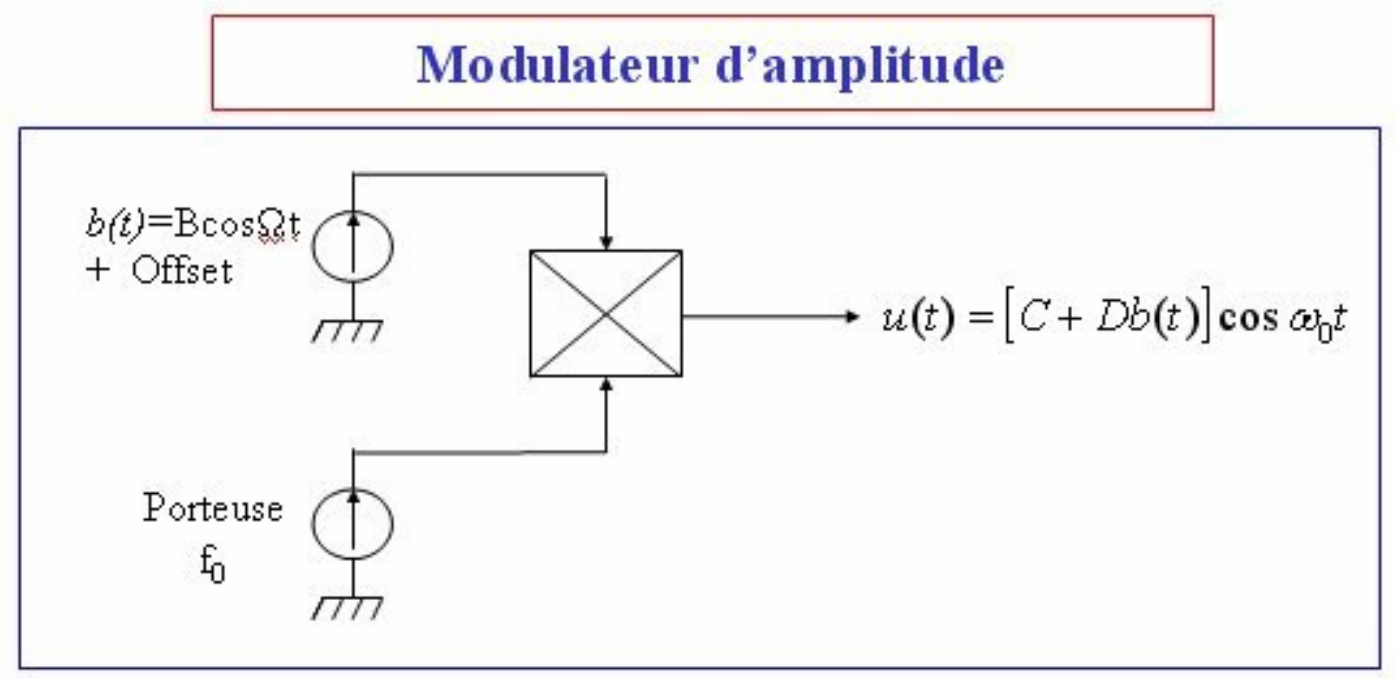

Fig. 18. Schéma du modulateur d'amplitude.

L'offset réglable permet d'obtenir un taux de modulation $\mathrm{m}$ variable de 0 à l'infini. Une modulation MDP-2 pourra être réalisée en prenant pour $b(t)$ un signal carré à moyenne nulle, c'est-àdire avec offset $=0$. Les symboles binaires associés sont :

$$
\begin{array}{ll}
a_{k}=1 & \text { si } b(t)>0 ; \\
a_{k}=-1 & \text { si } b(t)<0 .
\end{array}
$$

L'étude qui suit porte sur les 3 points suivants :

- la détection synchrone ;

- la boucle à verrouillage de phase ;

- la boucle de Costas.

Pour chacun de ces trois cas, le signal modulé est celui obtenu à l'aide du montage de la figure 18.

\section{C.1. La détection synchrone}

La fréquence de la porteuse est fixée à $30 \mathrm{kHz}$. La fréquence du signal modulant b(t) est fixée à $200 \mathrm{~Hz}$. Le $\theta_{0}$

déphasage de la référence $\mathrm{r}(\mathrm{t})$ simulée pour la détection synchrone est obtenue par un déphaseur $\mathrm{RC}$ à $\mathrm{AOP}$ cablé séparément sur plaque à bornes (Fig. 19). Le schéma du montage est représenté sur la figure 20.

Fig. 19. Déphaseur à amplificateur opérationnel.

\section{Circuit déphaseur}

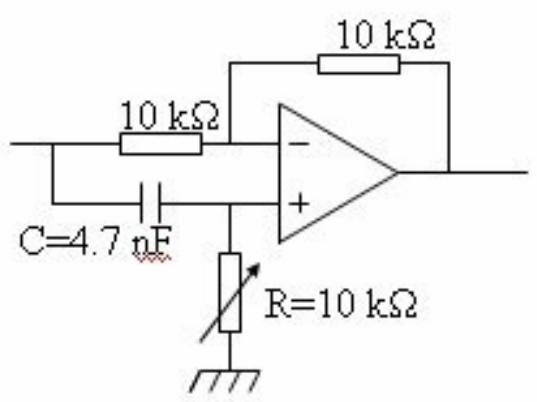




\section{Schéma d'analyse de détection synchrone}

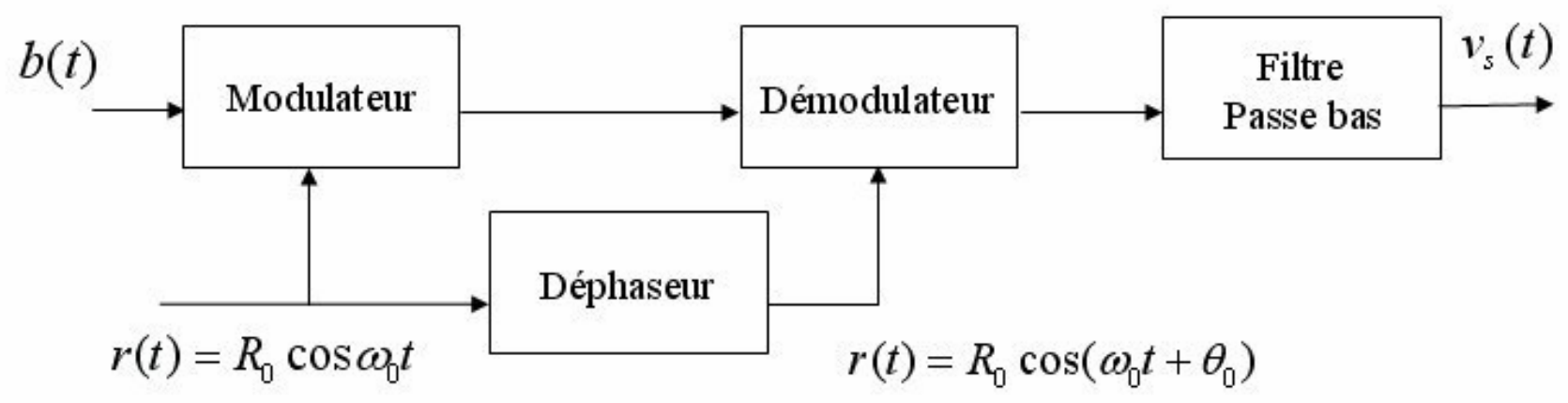

Fig. 20. Schéma du montage intégrant le déphasage de la porteuse.

- Pour $\theta_{0}=0$, observer la sortie démodulée $\mathrm{v}_{\mathrm{s}}(\mathrm{t})$.

- Vérifier que le signal est toujours correctement démodulé pour $m$ variant de 0 à l'infini.

- Vérifier l'influence de la phase ${ }^{\theta_{0}}$ sur l'amplitude du signal de sortie $\mathrm{v}_{\mathrm{s}}(\mathrm{t})$.

\section{C.2. La boucle à verrouillage de phase}

Cabler une PLL à l'aide du multiplieur M2 et de l'OCT de la maquette. Le filtre de boucle est un filtre RC passebas avec $R=10 \mathrm{k} \Omega$ et $\mathrm{C}=33 \mathrm{nF}$. On prendra un taux de modulation m inférieur à 1 pour le signal d'entrée $u(t)$.

- Mesurer la fréquence $\mathrm{f}_{0}$ de l'OCT pour une commande ve $=0$.

- Ajuster la fréquence de la porteuse du signal modulé à cette valeur de $\mathrm{f}_{0}$.

- Vérifier l'accrochage de la boucle.

- Mesurer le déphasage entre l'entrée $u(t)$ et la sortie OCT.

- Mesurer la plage de verrouillage.

- Observer le comportement de la boucle lorsque le taux de modulation $\mathrm{m}$ devient plus grand que 1.

- Utiliser une détection synchrone (avec M3) pour récupérer le signal utile. Étudier le comportement de l'ensemble en fonction de $\mathrm{m}$.

\section{C.3. La boucle de COSTAS}

Réaliser le cablage de la boucle de Costas. Les 2 filtres passe-bas sont de type $\mathrm{RC}$ avec $R=10 \mathrm{k} \Omega$ et $\mathrm{C}=33$ $\mathrm{nF}$. Puisqu'on ne s'intéresse qu'à la stabilité de la boucle, on prendra comme filtre de boucle une liaison directe de gain 1 .

- Effectuer l'accrochage sur signaux MDP+Porteuse avec $\mathrm{m}<1$.

- Étudier l'accrochage de la boucle lorsque $m$ tend vers l'infini.

- Sur signal modulant $b(t)$ sinusoïdal.

- Sur signal modulant $b(t)$ carré simulant une MDP-2.

- Comparer le signal démodulé à celui d'origine. 